Objectives: In the present study, volumes of the hippocampus were examined to identify the responsible lesions for neurobehavioral changes in CPNB, and patterns of atrophy in the hippocampus and brainstem were compared between patients with CPNB and AD.

Methods: The subjects were 32 patients, including 13 with CPNB ( 11 males and 2 females, age $51.2 \pm 12.1$ years old [mean \pm SD] $), 13$ with Behçet's disease without NB (non-NB) (10 males and 3 females, age $54.4 \pm 11.4$ years old), and 6 with AD (5 males and 1 female, age $78.8 \pm 7.5$ years old). All patients with BD satisfied the international classification criteria for BD. CPNB was defined as intractable, slowly progressive neurobehavioral changes and/or cerebellar ataxia accompanied by persistent elevation of interleukin-6 of $>20 \mathrm{pg} / \mathrm{mL}$ in cerebrospinal fluid on two different occasions at an interval of at least 2 weeks. All patients with AD satisfied Diagnostic and Statistical Manual of Mental Disorders (DSM)-IV criteria. Brain magnetic resonance imaging (MRI) was obtained from each subject. The areas of the midbrain tegmentum and pons were measured on mid-sagittal sections of T1-weighted images using image analysis software (Image $\mathrm{J}$ ver.1.45: $\mathrm{NIH}$, USA). Severity of gray matter loss in the hippocampal region and whole brain were investigated using Voxel-Based Specific Regional Analysis System for Alzheimer's Disease (VSRAD) software (Eisai Co., Ltd) to determine the degrees of hippocampal region atrophy (Z score) and whole-brain atrophy (WBAI). Thus, the $1 / Z$ score is positively correlated with the hippocampus volume. The ratio of the degree of brainstem atrophy to that of hippocampal atrophy was evaluated by the brainstem area value divided by the $1 / Z$ score (BA/H score) in each patient. Results: The brainstem area was significantly decreased in CPNB $(461.8 \pm 87.3$ [mean $\pm \mathrm{SD}$ ]) compared with that in AD $(661.9 \pm 56.1)$ and non-NB (666.1 \pm 50.6$)$ (Figure $A)$. VSRAD analysis showed that $Z$ score was significantly increased in CPNB $(1.46 \pm 0.70)$ and $A D(3.13 \pm 1.21)$ compared with that in non-NB $(0.77 \pm 0.40)$ (Figure B). Of note, the BA/H score, reflecting the brainstem/hippocampus volume ratio, was much lower in CPNB than in $A D[663.5 \pm 311.8$ vs $2018 \pm 667.6, p=0.001]$ (Figure C).
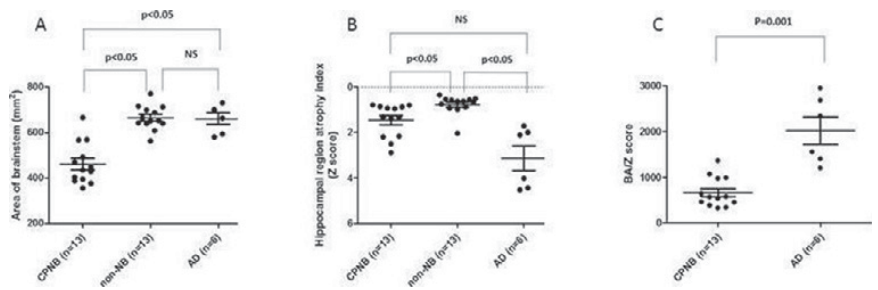

Conclusions: These results indicate that the hippocampus, in addition to the brainstem, is a common target lesion in CPNB, and this accounts for the progressive neurobehavioral dysfunction in this disease. Moreover, the data emphasize that brainstem atrophy is disproportionately greater than hippocampal atrophy in CPNB, in contrast to AD.

Disclosure of Interest: None declared

DOI: 10.1136/annrheumdis-2017-eular.1207

\section{THU0295 CLINICAL FEATURES OF TAKAYASU'S ARTERITIS FROM AN INCEPTION COHORT: EARLY DISEASE IS CHARACTERIZED BY "SYSTEMIC INFLAMMATION"}

F. Alibaz-Oner on behalf of Turkish Takayasu Arteritis Study Group. Rheumatology, Marmara University, School of Medicine, Istanbul, Turkey

Background: There is only retrospective and very limited data for the long term prognosis of Takayasu's Arteritis (TAK), a rare large-vessel vasculitis. In this study, we aimed to present the preliminary results of a Takayasu Inception Cohort settled for long term, prospective follow-up of only newly-diagnosed patients with TAK.

Methods: Patients fulfilling the American College of Rheumatology 1990 criteria for TAK and diagnosed in the last 12 months were included to the study. Patients' data were recorded in an electronic database of an international "Takayasu's Arteritis Registry" requiring baseline and at least annual visits. Data is compared with an historical Turkish cohort previously published (Bıçakçıgil, et al, 2009).

Results: The study included 128 patients (age: $38.9 \pm 13.1$ years, F/M: 112/16) with TAK from 15 tertiary Rheumatology centers in Turkey. The mean symptom duration of patients was 5.2 years at diagnosis. According to the angiographic

Table 1. Clinical characteristics of Inception and Retrospective Cohorts from Turkey

\begin{tabular}{lcc}
\hline & $\begin{array}{c}\text { Inception Cohort } \\
(\mathrm{n}=128)\end{array}$ & $\begin{array}{c}\text { Retrospective Cohort (Bıçakçıgil et al.) } \\
(\mathrm{n}=248)\end{array}$ \\
\hline Constitutional symptoms & $91 / 126(72.2 \%)$ & $163 / 248 /(66 \%)$ \\
Limb claudication & $66 / 106(62.3 \%)$ & $119 / 248(48 \%)$ \\
Carotidynia & $26 / 104(25 \%)$ & - \\
Pulseless & $37 / 104(35.6 \%)$ & $218 / 248(88 \%)$ \\
Musculoskeletal manifestations & $66 / 124(53.2 \%)$ & $104 / 248(42 \%)$ \\
Mucocutaneous manifestations & $32 / 121(26.4)$ & $22 / 248(8.8 \%)$ \\
Respiratory manifestations & $40 / 122(32.8 \%)$ & $22 / 184(12 \%)$ \\
Neurologic manifestations & $58 / 124(46.8 \%)$ & $156 / 248(63 \%)$ \\
Cardiac involvement & $23 / 106(21.7 \%)$ & $141 / 248(57 \%)$ \\
Ophthalmologic involvement & $29 / 125(23.2 \%)$ & $57 / 248(36 \%)$ \\
\hline
\end{tabular}

classification, $59.2 \%$ of the study group had type I and only $17.2 \%$ had type $\mathrm{V}$ disease. When we compared our results to our retrospective cohort (previously published by Turkish Takayasu Arteritis Study Group), constitutional symptoms (72.2\% vs $66 \%$ ) and limb claudication $(62.3 \%$ vs $48 \%$ ) were observed to be more frequent, whereas pulselessness ( $35.6 \%$ vs $88 \%$ ) was less in the inception cohort.(Table 1) Carotidynia was present only in the inception cohort. Similarly, mucocutaneous symptoms also seem to be a feature of newly-diagnosed disease $(26.4 \%$ vs $8.8 \%)$. Regarding comorbidities at diagnosis, the rate of dyslipidemia was $22 \%$, diabetes mellitus $6 \%$, smoking $28.5 \%$ and obesity $(\mathrm{BMI}>30) 15.8 \%$ among TAK patients. All patients were given oral corticosteroid (CS) therapy $(0.5-$ $1 \mathrm{mg} / \mathrm{kg}$ ) at diagnosis, 10 patients $(7.8 \%)$ also having CS pulses. In addition to CSs, 55 patients (43\%) were given methotrexate, 14 patients $(11 \%)$ azathioprine and $5(4 \%)$ cyclophosphomide at disease-onset.

Conclusions: Our results suggest that, in an inception cohort, signs and symptoms of "systemic inflammation" is more prominent in newly-diagnosed TAK patients, whereas vascular extent and damage accumulates during the disease course. The long term follow-up of our inception cohort will better show the actual course and predictors of prognosis in TAK.

Disclosure of Interest: None declared

DOI: 10.1136/annrheumdis-2017-eular.3856

\section{THU0296 THE ROLE OF ULTRASOUND IN THE MANAGEMENT OF GIANT CELL ARTERITIS (GCA) IN ROUTINE CLINICAL PRACTICE}

S. Monti ${ }^{1,2}$, A. Floris ${ }^{3}$, C. Ponte ${ }^{4}$, S. Vaggers ${ }^{2}$, W.A. Schmidt ${ }^{5}$,

A.P. Diamantopoulos ${ }^{6}$, C. Pereira ${ }^{2}$, R. Luqmani ${ }^{2} .{ }^{1}$ Rheumatology, University of Pavia, IRCCS Policlinico San Matteo Foundation, Pavia, Italy, Pavia, Italy;

${ }^{2}$ NDORMS, Rheumatology Department, Nuffield Orthopaedic Centre, University of Oxford, Oxford, United Kingdom; ${ }^{3}$ Rheumatology, University Clinic and AOU of Cagliari, Cagliari, Italy; ${ }^{4}$ Rheumatology, Hospital de Santa Maria, Rheumatology research Unit Instituto de Medicina Molecular, Lisbon Academic Medical Centre, Lisbon, Portugal; ${ }^{5}$ Rheumatology, Immanuel Krankenhaus Berlin, Medical Centre for Rheumatology Berlin-Bu, Berlin, Germany; ${ }^{6}$ Rheumatology, Hospital of Southern Norway Trust, Kristiansand, Norway

Background: Colour Doppler sonography (CDS) is an emerging diagnostic tool for giant cell arteritis (GCA), however, its use in routine practice is still not widespread.

Objectives: To develop a protocol using CDS and explore its value in the routine care of patients with GCA.

Methods: We developed a structured scanning protocol for CDS of temporal and axillary arteries (total of 8 anatomical sites scanned per patient) based on previously published methods. We tested the protocol on consecutive patients referred to a single rheumatology centre, with suspected or established GCA, between July 2014 and September 2016. We defined a positive scan by the presence of halo in at least one branch of a temporal artery (TA) or one axillary artery (AX). We report data from the first 293 consecutively scanned cases.

Results: We assessed 293 patients (mean age $72 \pm 10$, female/male 196/97), of whom 118 had clinically confirmed GCA. Amongst new referrals with confirmed diagnosis of GCA, $44 \%$ had a positive scan; two patients with a positive scan did not have GCA. $82 \%$ of new referrals patients showed exclusive TA involvement, $25 \%$ concomitant AX, and $4 \%$ exclusive AX involvement. High-dose glucocorticoid therapy had already been started in $78 \%$ of these patients for an average of $17 \pm 33$ days. Amongst this group, the sensitivity of CDS was $46 \%(95 \%$ IC $37 \%>55 \%)$, specificity $98 \%(95 \% \mathrm{Cl} 93 \%>99.8 \%)$, positive predictive value $96 \%$ $(88 \%>99.6 \%)$, and negative predictive value $60 \%$ (95\% IC $52 \%>68 \%$ ). During the period of observation, the rate of temporal artery biopsies (TAB) decreased significantly from $42 \%$ to $25 \%(p=0.002)$. During follow up, CDS was positive in $21 \%$ of 89 routine scans in asymptomatic individuals, compared to $58 \%$ in patients with confirmed clinical flares $(45 \%$ of whom had negative inflammatory markers). Over time, the number of halos per patient reduced; only new or flaring patients showed a halo in $>4$ sites. Halo size at the TA did not change significantly (average thickness $0.6 \pm 0.1 \mathrm{~mm}$ ), however, the size of AX artery halos significantly reduced from first referral $(1.6 \pm 0.4 \mathrm{~mm})$ to follow up $(1.4 \pm 0.2, p=0.01)$ or during subsequent flares $(1.4 \pm 0.2, p=0.02)$.

Conclusions: We have developed and tested a standardised methodology for CDS evaluation of GCA. CDS provides a high positive predictive value for a diagnosis of GCA in unselected patients from routine clinical practice, although prior high dose glucocorticoid therapy is likely to reduce its sensitivity. CDS allows for a significant reduction of TAB. We explored the role of CDS to detect disease flares and demonstrated a significant reduction in the extent of abnormalities, and of the size of halo of the AX arteries during follow up or flares. These findings could have a significant impact on the management of patients with suspected and confirmed GCA.

Disclosure of Interest: None declared

DOI: 10.1136/annrheumdis-2017-eular.4063 